\title{
Patch-clamp analysis of mutant voltage-gated sodium channels causing congenital insensitivity to pain
}

Kenkichi Kiyosawa ${ }^{1,2}$, Yuki Sugiyama1 ${ }^{\text {, Toshihide Kashihara }}{ }^{2}$, Tsutomu Nakada ${ }^{2}$, Mitsuhiko Yamada ${ }^{2}$, Mikito Kawamata $^{1}$ ${ }_{1}$ Department of Anesthesiology and Resuscitology, Shinshu University School of Medicine

${ }_{2}$ Department of Molecular Pharmacology, Shinshu University School of Medicine

Figure.
A

D

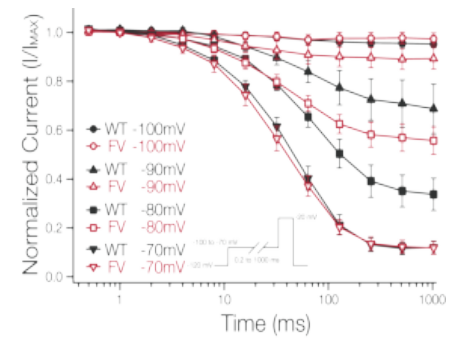

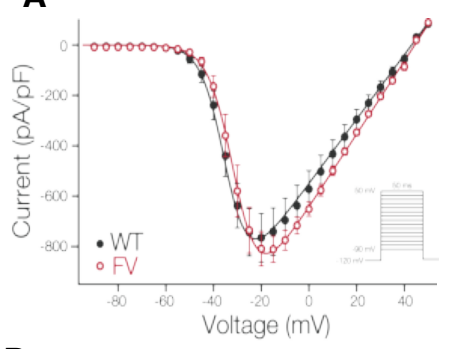

Background and Goal of Study:

Congenital insensitivity to pain (CIP) is a rare disease associated with a loss of pain experience. We found a 73-yearold female who was clinically diagnosed to suffer from congenital insensitivity to pain. She had normal intelligence, motor function, but often experienced thermal injuries and bone fractures, because she felt less heat and pain (Table1, 2). We thus carried out a genetic analysis and identified a new mutation in SCN9A gene. Goal of this study is to analyze the biophysical properties of the mutant $\mathrm{Na}_{\mathrm{V}} 1.7$ channels.

\section{Materials and Methods:}

Patient. The patient is a 73-year-old female. After approval of a study protocol by the ethical committee of our institution, informed consent was obtained from the patient.

Physiological test. As for neurophysiological function, we examined peripheral nerve conduction, quantitative sensory testing using CASE IV (WR Medical Electronics, MN, USA). Exon screening. Her blood samples were collected for genetic analysis. We detected a novel homozygous missense mutation in a SCN9A gene encoding $\mathrm{Na}_{\mathrm{V}} 1.7$ channels.

Electrophysiology. The mutated $\mathrm{Na}_{\mathrm{V}} 1.7$ (FV) and wild-type $\mathrm{Na}_{\mathrm{V}} 1.7$ (WT) were subcloned into pcDNA3 and transfected into a human embryonic kidney cell line, tsA201 cells to analyze its electrophysiological properties with the patch-clamp method.

\section{Conclusion(s):}

This study suggests that the decreased overlap of the activation and inactivation curves results in the decreased ramp current, which would in turn cause hypo-excitability of dorsal root ganglion neurons and insensitivity to pain in this patient
C

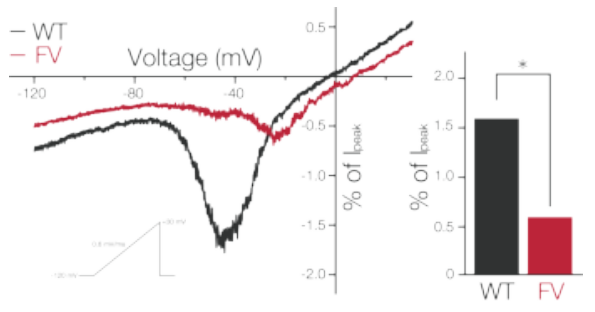

$\mathbf{F}$

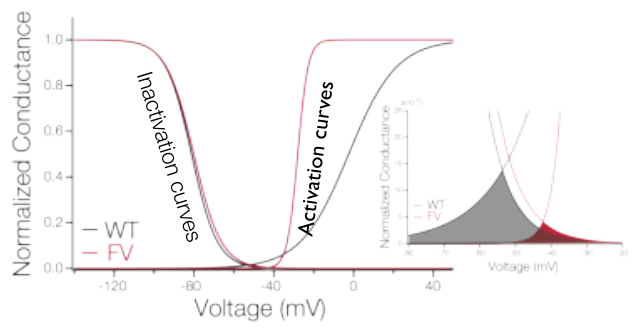

Results:

Neurophysiological function analysis

Table 1. Quantitative sensory testing: QST

\begin{tabular}{cc}
\hline QST & Percentile of normal control \\
\hline Heat & $99.6 \%$ \\
Cold & $73.0 \%$ \\
Vibration & $96.0 \%$ \\
\hline
\end{tabular}

Table 2. Quantitative sensory testing: QST

\begin{tabular}{cccc}
\hline \multicolumn{3}{c}{ Site } & \multicolumn{2}{c}{ Velocity } \\
\hline Motor & Median nerve & $54.6 \mathrm{~m} / \mathrm{s}$ & $(58.4 \pm 4.4 \mathrm{~m} / \mathrm{s})$ \\
Sensory & Median nerve & $52.1 \mathrm{~m} / \mathrm{s}$ & $(57.8 \pm 6.0 \mathrm{~m} / \mathrm{s})$ \\
& Sural nerve & $50.0 \mathrm{~m} / \mathrm{s}$ & $(57.5 \pm 3.0 \mathrm{~m} / \mathrm{s})$ \\
\hline
\end{tabular}

Electrophysiological analysis

We examined the voltage dependence of activation and steadystate fast inactivation. FV exhibited a significant depolarizing shift in channel activation compared with WT (Fig. A), whereas FV and WT exhibited a similar voltage-dependency of the fast inactivation (Fig. B). However, FV exhibited significantly faster recovery from inactivation than WT (Fig. E). We also compared the currents of ramp depolarizations (Fig. C). Interestingly, FV had the significantly smaller ramp current amplitude than WT as assessed with either 0.2 or $0.6 \mathrm{mV} / \mathrm{ms}$ from -120 to $+30 \mathrm{mV}$. The peak ramp current amplitude $(0.6 \mathrm{mV} / \mathrm{ms})$ normalized to that evoked by a voltage step to $-20 \mathrm{mV}$ was $2.34 \pm 0.27 \%$ for WT $(n=4)$ vs. $0.90 \pm 0.40 \%$ for $F V(n=4)(P<0.05)$. The depolarizing shift of fast inactivation, but the more depolarizing shift of activation for FV, compared with WT results in a substantially decreased window current (Fig. F). 